\title{
Observational evidence of the primary role played by photospheric motions in magnetic helicity transport before a filament eruption
}

\author{
P. Romano ${ }^{1}$, L. Contarino ${ }^{2}$, and F. Zuccarello ${ }^{1}$ \\ ${ }^{1}$ Dipartimento di Fisica e Astronomia - Sezione Astrofisica, Università di Catania, via S. Sofia 78, 95125 Catania, Italy \\ e-mail: promact.astro.it \\ 2 INAF - Osservatorio Astrofisico di Catania, via S. Sofia 78, 95125 Catania, Italy
}

Received 6 August 2004 / Accepted 20 November 2004

\begin{abstract}
Many filament eruptions can be suitably described in the framework of the kink instability model, although it is not always easy to discriminate whether the helical flux rope writhes due to new emerging flux or to photospheric horizontal motions. In this paper we provide observational evidence of the important role which can be played by horizontal motions in filament instability and eruption. More precisely, we describe the analysis of the eruption of a reverse-S-shaped filament associated with a flare of class M6.3, that occurred on 15 June, 2001 in the active region NOAA 9502. Using TRACE $195 \AA$ images we studied the morphological evolution of the EUV filament channel. Using 1 minute cadence MDI full-disc longitudinal magnetograms we analyzed the magnetic evolution of the entire active region. The geometrical parameters of the EUV filament channel and the horizontal velocities in the areas corresponding to the filament footpoints were determined and agreed with the kink instability. Moreover, the analysis of MDI magnetograms showed that a sudden and strong increase in the magnetic helicity transport rate to the corona preceded and accompanied the filament eruption. During the same time interval, on the one hand the emergence of magnetic flux in both polarities became negligible, but on the other hand the velocity pattern at the filament ends showed horizontal, counterclockwise motions, which could make a significant contribution to the transformation, from twist to writhe, of the magnetic helicity accumulated along the filament before its eruption. This result seems to indicate that in this event the transport of magnetic helicity exceeding the limit for the kink instability is primarily due to photospheric motions, while the contribution from the emerging flux is negligible.
\end{abstract}

Key words. Sun: activity - Sun: magnetic fields - Sun: filaments

\section{Introduction}

Many phenomena of sudden magnetic energy release occurring in the solar atmosphere, like flares, coronal mass ejections and erupting filaments, are characterized by topological complexity of the magnetic field. Filaments often manifest this topological complexity by means of a helical-like pattern, which becomes more visible during their eruption (see, e.g., Romano et al. 2003a). Although helical flux ropes are clearly ejected by the Sun, there is still some controversy on whether they form below the solar atmosphere or if the structures get more and more twisted because of horizontal motions and/or emergence of new magnetic flux just before their ejection.

Martin (1998) found that filament patterns exhibit hemispheric preference independently of the solar cycle: left-handed features preferentially appear in the northern hemisphere and right-handed features in the southern.

A quantitative measure of the chiral properties of filaments is the magnetic helicity $H_{\mathrm{m}}=\int_{V} \boldsymbol{A} \cdot \boldsymbol{B} \mathrm{d} V$, where $\boldsymbol{A}$ is the magnetic vector potential and $\boldsymbol{B}=\nabla \times \boldsymbol{A}$ is the magnetic field
(Berger 1984). To date there is no consensus on whether the observational determinations of right and left handed signatures of the chirality of filaments correspond to positive and negative signs of twist helicity, respectively, or to the inverse (Martin 2003). However, a simple and direct way of identifying the helicity of the axial component of filaments has been proposed by Rust (2003), who suggested that $\boldsymbol{S}$-shaped filaments, like $S$-shape sigmoids, are evidence of positive helicity, and reverse $S$-shaped ones of negative helicity.

Magnetic helicity is a useful quantity because it is conserved in closed volumes, even in resistive MHD (Taylor 1986). Since the solar corona is an open volume having the photosphere as a boundary with vertical flux, the magnetic helicity can be transported across the boundary by velocity fields in the photosphere (Moon et al. 2002). Following Berger \& Field (1984), the Poynting theorem for magnetic helicity gives:

$\frac{\mathrm{d} H}{\mathrm{~d} t}=\oint 2\left(\boldsymbol{B} \cdot \boldsymbol{A}_{\mathrm{p}}\right) v_{\mathrm{n}} \mathrm{d} S+\oint-2\left(\boldsymbol{v} \cdot \boldsymbol{A}_{\mathrm{p}}\right) B_{\mathrm{n}} \mathrm{d} S$ 
where $\boldsymbol{A}_{\mathrm{p}}$ is the vector potential of the potential magnetic field $\boldsymbol{B}$, which is solely specified by the observed flux distribution on the photosphere with the equations:

$\nabla \times A_{\mathrm{p}} \cdot z=B_{\mathrm{n}}, \quad \nabla \cdot A_{\mathrm{p}}=0, \quad A_{\mathrm{p}} \cdot z=0$,

with $B_{\mathrm{n}}$ and $v_{\mathrm{n}}$ components of the magnetic and velocity fields normal to the photosphere. Therefore the magnetic helicity is a valid tool for measuring how the magnetic field is sheared or twisted in the corona, compared to its lowest energy state (potential field). Equation (1) shows that the magnetic helicity in the corona can change either by passage of twisted (or sheared) fields through the photosphere (first term) or by horizontal motions twisting (or shearing) magnetic fields (second term).

Chae (2001) devised a direct way of deriving the second term from line-of-sight magnetograms of SOHO/MDI using the local correlation tracking (LCT) method developed by November \& Simon (1988). Recently, Kusano et al. (2002) have estimated both terms of Eq. (1) using the vertical velocity component indirectly determined from the induction equation. However, more recently Demoulin \& Berger (2003) have noted that the LCT method does not measure the plasma motions, but rather the displacement of the photospheric footpoints of magnetic flux tubes. This displacement is the result of both horizontal and vertical plasma motions. More precisely, using the velocity deduced from the LCT method, we get the full helicity flux and it is not correct to add the helicity flux associated to vertical plasma motions to the helicity flux deduced from the velocities of photospheric footpoint motions.

A study of the relationship between magnetic helicity transport and filament activation has been carried out by Romano et al. (2003a): applying the LCT method to line-of-sight magnetograms of SOHO/MDI at the footpoints of an active filament, they found a strong correlation between filament eruptions and helicity transport rate, although the amount of helicity change due to shearing motions was not sufficient to reach a critical value of the screw of the magnetic field determined by the kink instability theory. However, the horizontal motions may still provide a significant contribution to instability in other events where the magnetic helicity transport from photosphere to corona is more evident, as in the formation and activation of filaments characterized by a sigmoid shape.

For this reason, in this paper we focus our attention on the eruption of a kinked filament, related to an M6.3 class flare and characterized by a reverse- $\boldsymbol{S}$ shape. We studied its activation phase, measuring the geometrical parameters observed in the EUV filament channel visible in TRACE images at $195 \AA$. We also computed the photospheric velocity field and the helicity transport rate from line-of-sight magnetograms of SOHO/MDI, using the method proposed by Chae (2001). Our results indicate that photospheric shearing motions do play an important role in the transport of negative magnetic helicity exceeding the limit for the kink instability.

\section{Observations}

A filament eruption associated with a flare of GOES class. M6.3 occurred on 15 June, 2001 in the active region
NOAA 9502 (average latitude 26 South). The filament eruption occurred from 9:52 to 10:21 UT, while the flare was registered by GOES between 10:01 and 10:20 UT in the region of coordinates $26 \mathrm{~S} 41 \mathrm{E}$. In order to analyze this event we used images of the corona taken by TRACE at $195 \AA$ and full-disk line-of-sight magnetograms taken by MDI/SOHO.

TRACE images were taken in full resolution mode, i.e. $768 \times 768$ pixel, with a pixel size of 0.5 arcsec and a field of view of $6.4 \times 6.4$ arcmin. We considered images acquired on 15 June, from 4:59 to 9:00 UT with a time cadence of $30 \mathrm{~min}$ and from 9:00 to 11:30 with a time cadence varying between $3 \mathrm{~s}$ and $1 \mathrm{~min}$. The raw data were processed by means of the subtraction of the pedestal and dark current, as well as by using the correction for radiation spikes and hot pixels.

MDI images were recorded by a $1024 \times 1024$ CCD detector with a spatial resolution of 2 arcsec, covering a period of $35 \mathrm{~h}$, from 14 June, 2001 at 13:00 UT to 15 June at 23:59 UT, with a time cadence of $1 \mathrm{~min}$. The data set has 4 gaps (on 14 June, from 22:22 to 23:11 UT, on 15 June, from 04:03 to 04:11 UT, from 12:01 to $15: 45$ UT and from 20:18 to 20:26 UT). We analyzed a field of view of $300 \times 300$ arsec centered in the NOAA 9502.

All the data were aligned by applying the nonlinear mapping defined by the differential solar rotation (Howard et al. 1990), correcting the geometrical foreshortening due to the spherical geometry of the Sun, and by rotating images until 17 June at 16:00 UT, when the active region passed through the central meridian.

\section{Data analysis}

\subsection{Morphology and evolution}

On 15 June, 2001 the active region NOAA 9502 was characterized by four main sunspots with an intermediate population (Fig. 1a). Three sunspots, more or less aligned with the equator, had negative polarity, while the eastern one had positive polarity (Fig. 1b). Images of the same field of view taken at $195 \AA$ show that an EUV filament channel is located on the Eastern side of the active region, along the magnetic inversion line (Fig. 2).

The sequence of images reported in Fig. 3 shows the evolution of this EUV filament channel. At the beginning of the TRACE observations, at 04:59 UT (Fig. 3a), it was present with a smooth curved shape and its boundaries did not appear well defined, probably because the process of formation was not yet completed. Two hours before the eruption (Fig. 3b) the EUV channel assumed a clear reverse $\boldsymbol{S}$-shape, which is evidence of a negative helicity of the axial component of the filament, as noted by Rust (2003). Moreover, the EUV channel appeared overlapped by many loops connecting the opposite polarity sunspots. At 9:52 UT the activation phase of the filament started with a brightening slightly parallel to the EUV channel, which showed a more kinked shape, clearly visible in Fig. 3c. At 10:05 the filament started to rise faster than in the preceding phase, with an increase in the brightening on both sides (Fig. 3d). 


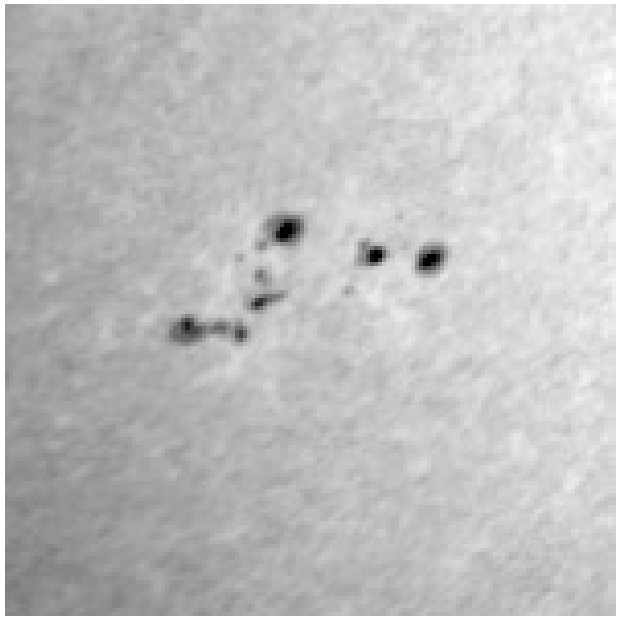

(a)

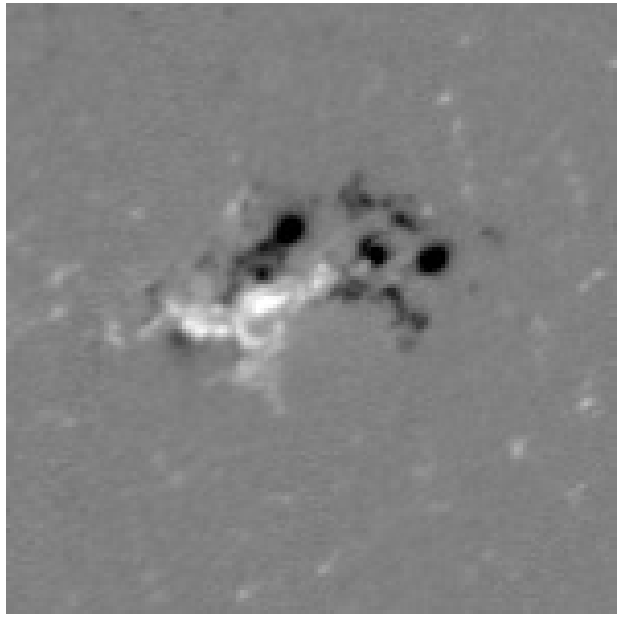

(b)

Fig. 1. Active region NOAA 9502 on 15 June, 2001, at 9:50 UT: a) MDI continuum image; b) MDI line-of-sight magnetogram. North is at the top, West to the right. The field of view is about $300 \times 300 \mathrm{arcsec}$.

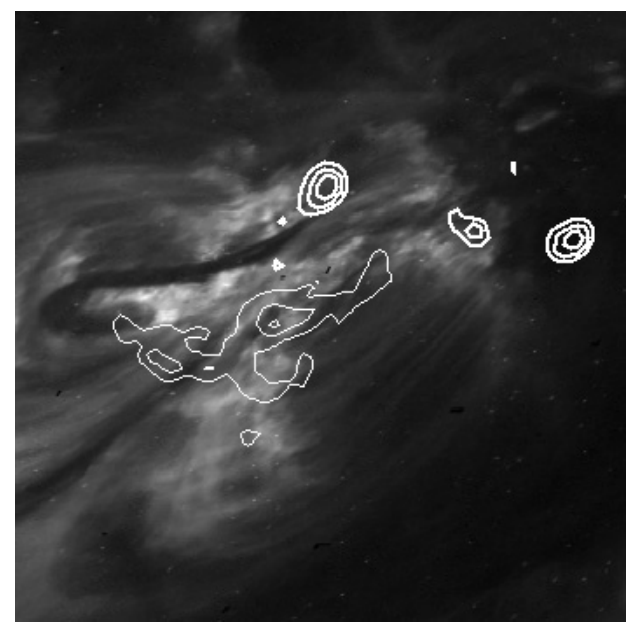

Fig. 2. $195 \AA$ image of NOAA 9502 taken by TRACE on 15 June, 2001, at 9:50 UT. The field of view is about $200 \times 200 \mathrm{arcsec}$. Thin and thick contours indicate positive and negative magnetic fluxes respectively. Contour levels are drawn at $+300,+500$ and $+700 \mathrm{G}$ for positive polarity and at $-500,-700$ and $-900 \mathrm{G}$ for negative polarity.

We identified the region occupied by the EUV filament channel as the one filled by the filament kinked flux rope. Following Rust \& Kumar's (1996) model we assumed that the flux rope was cylindrically symmetric before becoming kinked and that after the kink process it was still contained in a cylindrical curved region, anchored on both ends in the photosphere. We computed the values of the length $L$ and width $R$ of this region in a $195 \AA$ image acquired at 9:50 UT. To avoid errors due to perspective effects, we rotated TRACE images to correct for the solar differential rotation until 17 June at 16:00 UT, when the active region passed through the central meridian. We found that at the beginning of the activation phase $L \sim 70000 \mathrm{~km}$ and $R \sim 20000 \mathrm{~km}$.

During the filament eruption the EUV channel appeared to be composed of a bundle of helically twisted threads (see the arrow in Fig. 4a). From 10:07 UT the filament plasma was apparently thrown towards the West (see the arrow in Fig. 4b) and most of the active region became brighter due to the flare, which reached its peak at 10:13 UT, as reported by GOES. The filament eruption ended at 10:21 UT.

\subsection{Magnetic flux}

We calculated the temporal variations of the integrated positive and negative magnetic fluxes over the entire active region before, during, and after the event of interest. We increased the signal-to-noise ratio of the line-of-sight magnetic field maps by averaging every five successive magnetograms of $1 \mathrm{~min}$ cadence, and we also took into account that Berger \& Lites (2002) recently discovered that $\mathrm{SOHO} / \mathrm{MDI}$ underestimates the longitudinal magnetic field by a factor of $\sim 0.64$.

Figure 5 reports both the positive (thin line) and negative (thick line) magnetic flux, in absolute value, as a function of time. The origin in the abscissa corresponds to 13:00 UT, on 14 June. The interruptions in the magnetic flux profiles correspond to temporal gaps in MDI observations, the vertical lines indicate the beginning and the end of the event. At the beginning of the observations the integrated positive and negative magnetic fluxes in NOAA 9502 exhibited an average value of $7.5 \times 10^{21} \mathrm{Mx}$ and of $10.5 \times 10^{21} \mathrm{Mx}$, respectively. The difference of about $3 \times 10^{21} \mathrm{Mx}$ is probably due to the field of view considered, which did not include part of the surrounding flux. During the first 20 hours we can see an increase of about $0.7 \times 10^{21} \mathrm{Mx}$ in the positive magnetic flux and a decrease of about $1 \times 10^{21} \mathrm{Mx}$ in the negative magnetic flux. Although we corrected the data for the geometric projection, we have to consider that this trend is affected by the magnetic strength projection, which cannot be corrected because of the lack of vector magnetograms. Therefore, we must take into account that the fluxes in both polarities are underestimated and that this underestimation decreases while the active region moves from the East limb to the central meridian. However, apart from the projection effects, during the time interval starting two hours 


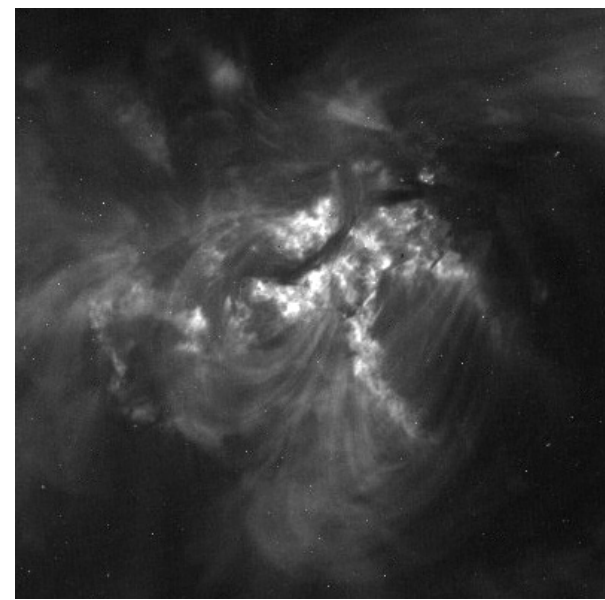

(a) 04:59:15 UT

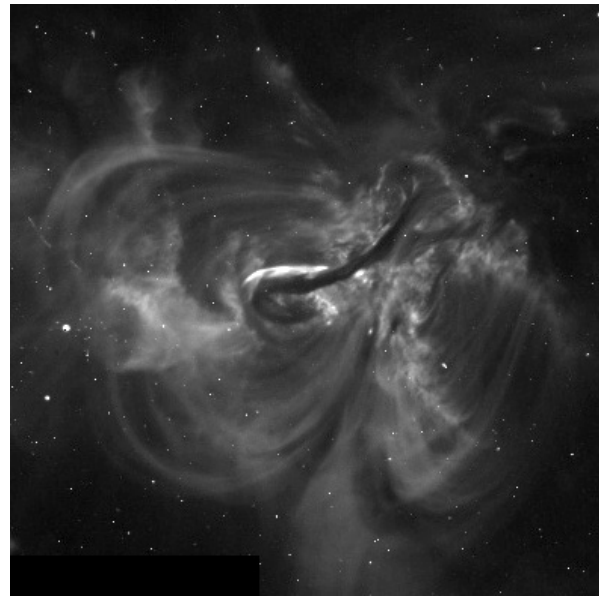

(c) 10:03:10 UT

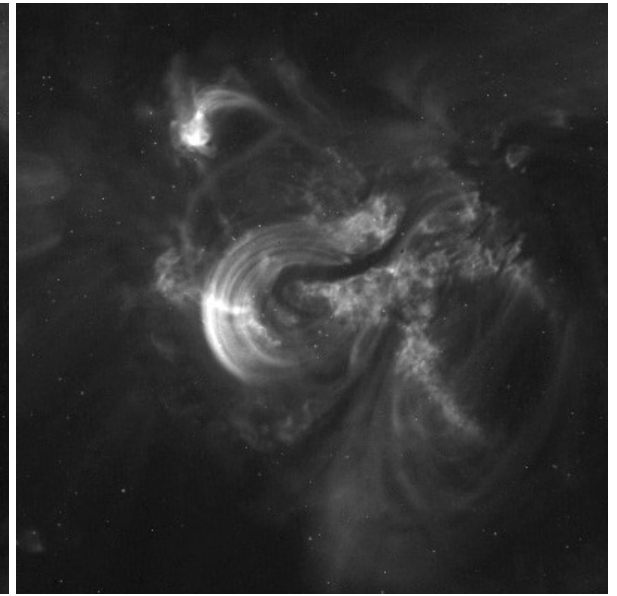

(b) 08:00:12 UT

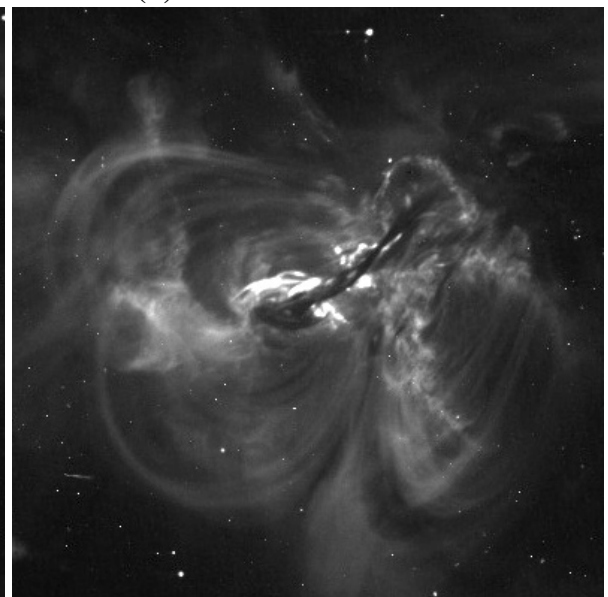

(d) 10:04:59 UT

Fig. 3. Images at $195 \AA$ A of the filament evolution before its eruption, as observed by TRACE on 15 June, 2001. The field of view of each image is about $300 \times 300$ arcsec.

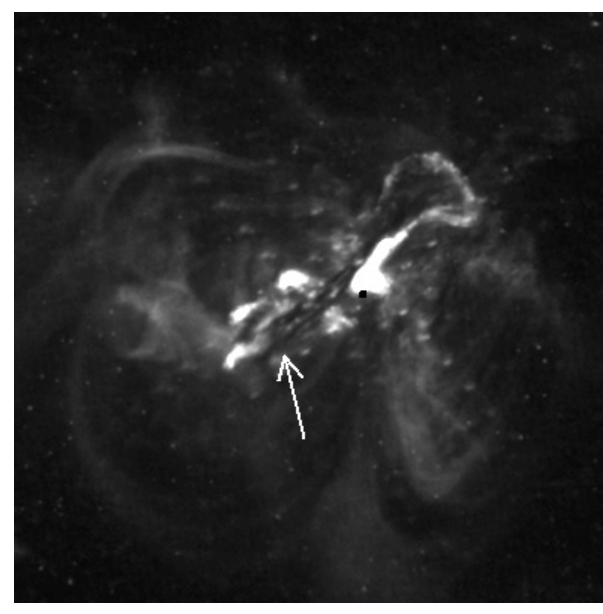

(a) 10:06:27 UT

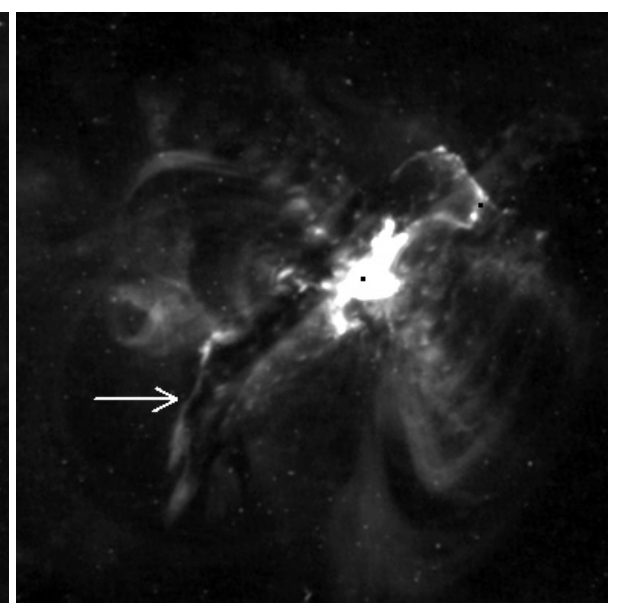

(b) 10:07:55 UT

Fig. 4. $195 \AA$ Amages of the filament eruption and of the consequent flare. The field of view is about $300 \times 300$ arcsec.

before the event till the end of the observational period, we note a change in the magnetic flux trend for both polarities. Moreover, after the event the positive and negative magnetic fluxes became comparable, and the difference of the magnetic fluxes at the end of the data set was less than $0.5 \times 10^{21} \mathrm{Mx}$.

\subsection{Velocity fields}

In order to determine the horizontal motions relevant to the event considered, we calculated the photospheric velocity field from the MDI magnetograms by using the local correlation 


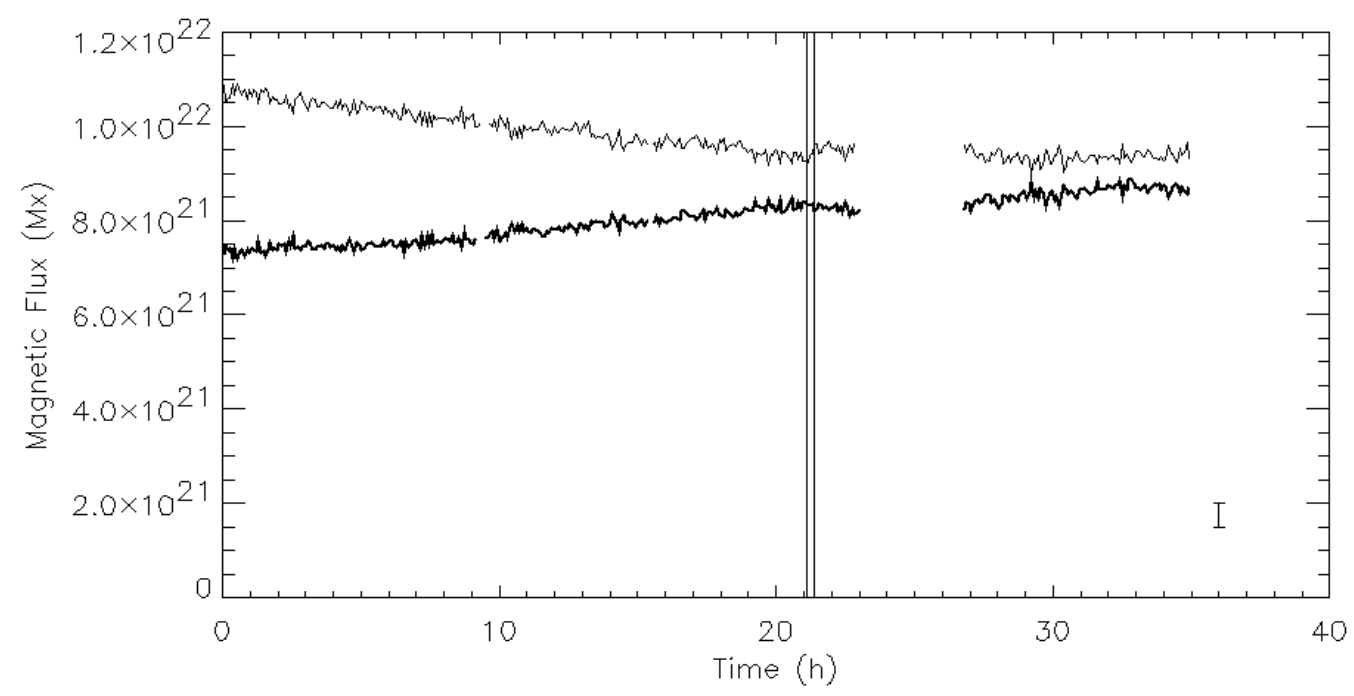

Fig. 5. Temporal magnetic flux variation for each polarity in NOAA 9502. The thin line and the thick line indicate positive and negative polarity, respectively. $t=0$ corresponds to 13:00 UT on June 14 . The error bar represents the rms of the signal. The vertical lines indicate the start and the end of the flare, as registered by GOES.

tracking (LCT) method (November \& Simon 1988). We considered a time interval of $20 \mathrm{~min}$ and chose the full width at half maximum of 8 arcsec (4 pixels). We set the velocity equal to zero when the magnetic flux was lower than $10 \mathrm{G}$. The obtained rms speed of magnetic concentrations is about $0.07 \mathrm{~km} \mathrm{~s}^{-1}$.

Figure 6 shows the maps of photospheric horizontal velocities in the active region before, during and after the eruption event. Circles A and B in Fig. 6a indicate the areas where the filament footpoints were located, as deduced from Fig. 2. Some hours before the filament eruption we noted peculiar motions along the magnetic inversion line. In particular at 9:20 UT we can see counter-clockwise motions in circles A and B (Fig. 6a). These rotating motions range from $0.2 \mathrm{~km} \mathrm{~s}^{-1}$ to $0.4 \mathrm{~km} \mathrm{~s}^{-1}$ and are more evident in circle A. This pattern is probably responsible for the reverse $S$-shape of the filament and becomes more and more evident till 10:25 UT (Fig. 6b), when the flare ended.

The flare main phase was also accompanied by a global variation of the velocity pattern due to a possible rearrangement in the magnetic field of the active region. In particular, we observed a strong variation in the horizontal motions in the area underlying the first brightening along the EUV filament channel (see circle $\mathrm{C}$ in Fig. 6b).

At the end of the eruption the velocity field assumed again the configuration observed before the event, although in the areas $\mathrm{A}$ and $\mathrm{B}$ the speed of the counter-clockwise motion decreased (Fig. 6c).

\subsection{Helicity transport}

In order to compute the magnetic helicity injected and accumulated in the active region from the photosphere to the corona, we used the procedure described by Chae (2001). The temporal variation in the magnetic helicity transport rate showed an amplitude of the noise of about $1 \times 10^{39} \mathrm{Mx}^{2} \mathrm{~h}^{-1}$. This kind of amplitude fluctuation was also noticed in previous studies (Chae 2001) and was attributed not only to noise, but also to intrinsic variations associated with surface convective flows.
Moreover, the use of full-disk magnetograms could also provide non-negligible effects in the application of the LCT. By selecting only the modes with periods longer than 1 hour, we constructed the low-pass-filtered signal. The convolution between the low-pass-filter and the signal of the magnetic helicity transport rate since 13:00 UT on 14 June is shown in Fig. 7. The standard deviation of the obtained signal is $1 \times 10^{38} \mathrm{Mx}^{2} \mathrm{~h}^{-1}$. During the first $17 \mathrm{~h}$ the magnetic helicity transport rate does not show a prevalent sign, but it seems to oscillate around zero. Moreover, we could determine that the first two negative variations correspond to two events registered by GOES: the former, of class C2.9, occurred from 20:04 to 20:24 on 14 June and the latter, of class C2.1, occurred from 1:59 to 2:18 on 15 June. At 6:00 UT, four hours before the filament eruption and the associated flare, we note a new strong variation in the transport rate: a prevalent negative magnetic helicity is transported to the corona with a rate peak of $-3.0 \times 10^{39} \mathrm{Mx}^{2} \mathrm{~h}^{-1}$ at 10:05 UT. We also note that at 10:20 UT, corresponding to the end of the flare as registered by GOES, the magnetic helicity transport rate has returned to its preflare value. After the observation gap, from 12:01 to 15:45 UT, the signal shows some instabilities of less intensity, and reaches a more stable trend at 17:00 UT.

Figure 8 reports the accumulated helicity change from the beginning of the observations. We note that the active region is characterized by a positive trend of accumulation of magnetic helicity during the first half of the observing run. Two hours before the start of the eruption there is an inversion of the sign of the helicity transport, while some tens of minutes before the event we can see a steep increase in the negative helicity to $-2 \times 10^{40} \mathrm{Mx}^{2}$. In only $90 \mathrm{~min}$, from 9:00 to 10:30 UT, $-4 \times 10^{40} \mathrm{Mx}^{2}$ are transported to the corona. Therefore we can divide the long-term change of the magnetic helicity into three phases: a first phase characterized by a gradual increase of the positive magnetic helicity, a steep decrease just before the filament eruption and a final, strong increase of the negative helicity transport. 


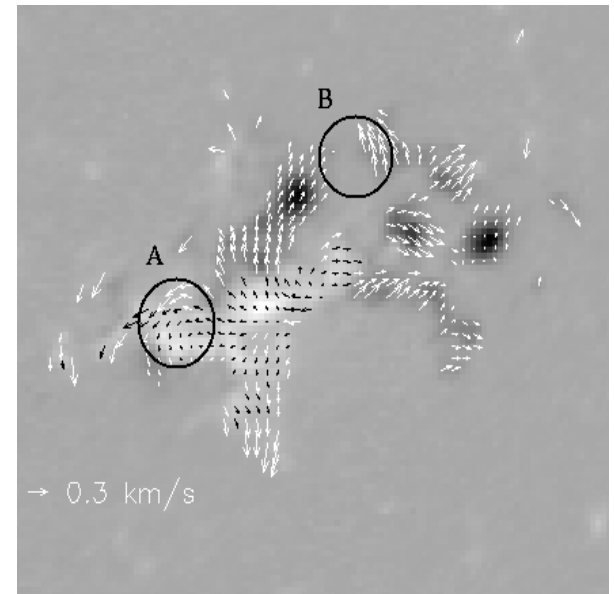

(a) 9:20 UT

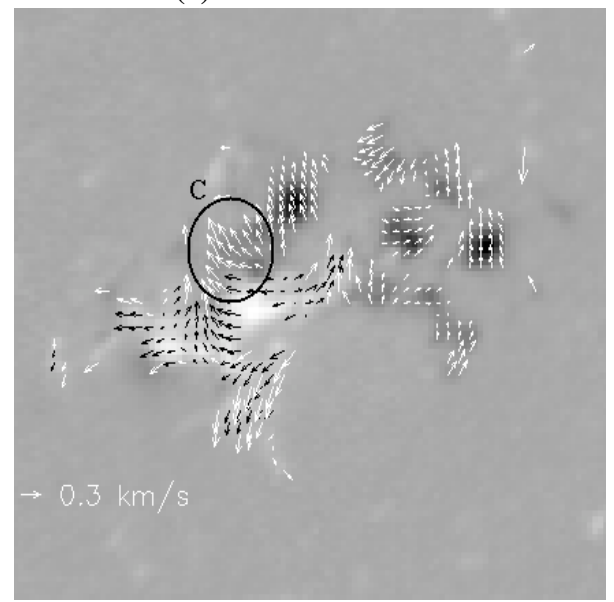

(b) 10:25 UT

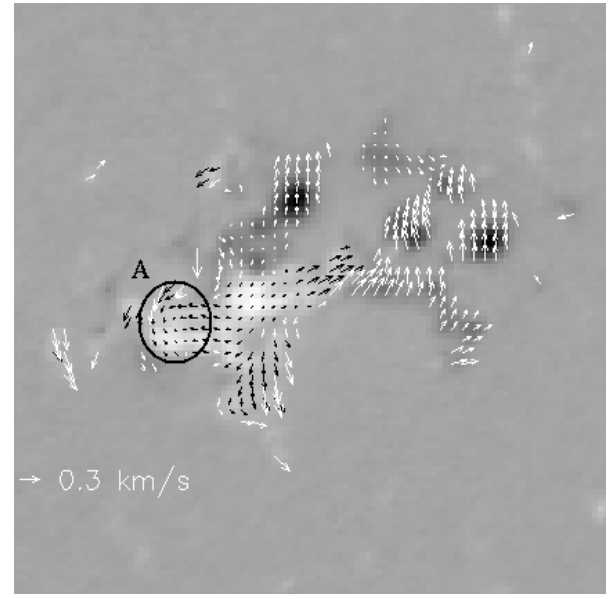

(c) 11:11 UT

Fig. 6. Vector maps of photospheric horizontal flows superimposed on MDI magnetograms obtained before, during and after the filament eruption. The field of view is about $200 \times 200$ arcsec, as in Fig. 2 . Circles A, B, C and D indicate areas characterized by peculiar motions.

\section{Discussion}

The magnetic helicity in filaments can be estimated by:

$H_{\mathrm{m}}=(T w+W r) \Phi^{2}$ where the twist $T w$ is the number of turns of the field around the axis, the writhe $W r$ is the number of times the axis loops on itself (i.e., it is the helicity of the axis itself) and $\Phi$ is the magnetic flux in the filament (Rust 2003). Both $T w$ and $W r$ can change due to the emergence of new magnetic flux or due to the horizontal motions of the photospheric intersections of the magnetic flux tubes.

Recently, Romano et al. (2003a), studying the relationship between filament eruptions and the injection of magnetic helicity in four different events, found that the emergence of magnetic flux was consistent with an amount of magnetic helicity transport sufficient to reach a critical value in the context of the kink instability. However, although observations show that flux ropes can emerge already twisted, it is also possible that the photospheric motions can drive the twist and the screw of the filament magnetic field. In particular, this latter mechanism could be efficient in filaments which show a sinuous morphology, like an $\boldsymbol{S}$ - or reverse $\boldsymbol{S}$-shape, where a twisted flux rope writhes because the amount of magnetic helicity in it exceeds the limit for the kink instability:

$2 \pi T_{w} R / L>0.581$

where $R / L$ is the ratio between the width of the $S$ and its length (Rust \& Kumar 1996).

In this context we considered the evolution of the reverse $S$-shape filament located in the active region NOAA 9502. At the beginning of the TRACE observations (4:59 UT), when the filament formation process was not yet complete, this already showed a curved shape, which indicated that the magnetic field emerged already twisted (see Low 1994). The sigmoid shape of the filament became more and more pronounced till the beginning of the filament activation, at 9:52 UT, when it assumed a clear reverse $S$-shape, corresponding to an amount of negative magnetic helicity exceeding the limit for the kink instability. Since the temporal evolution of the integrated magnetic flux did not show any significant variation before the eruption, we assume that part of this negative magnetic helicity was transported from the photosphere by the horizontal motions of the filament footpoints.

The vector maps of photospheric horizontal flows seem to confirm our hypothesis. In fact, we registered counterclockwise motions in both areas corresponding to the filament footpoints (Fig. 6a) some hours before the activation phase. Moreover, at 9:52 UT, when the first signatures of the filament eruption appeared, the ratio $R / L$ was $\sim 0.28$, which corresponds to $T w$ greater than 0.32 , exceeding the limit for the kink instability. Therefore, if we consider that the range of the peculiar motions measured at the filament footpoints was between $0.2 \mathrm{~km} \mathrm{~s}^{-1}$ and $0.4 \mathrm{~km} \mathrm{~s}^{-1}$ and that the diameter of the footpoints was equal to $5000 \mathrm{~km}$, we obtain a time to reach the twist limit ranging between 6.9 and $4.6 \mathrm{~h}$. This evolution time is comparable with the time spent to reach the instability of the filament, as observed in the TRACE sequence.

The movie at $195 \AA$ of the filament eruption showed the writhing of the filament, like many other events observed previously (Rust 2003). This rapid evolution, occurring in about $10 \mathrm{~min}$, is a further indication that the flux rope developed a kink instability. For this reason, using the velocity deduced 


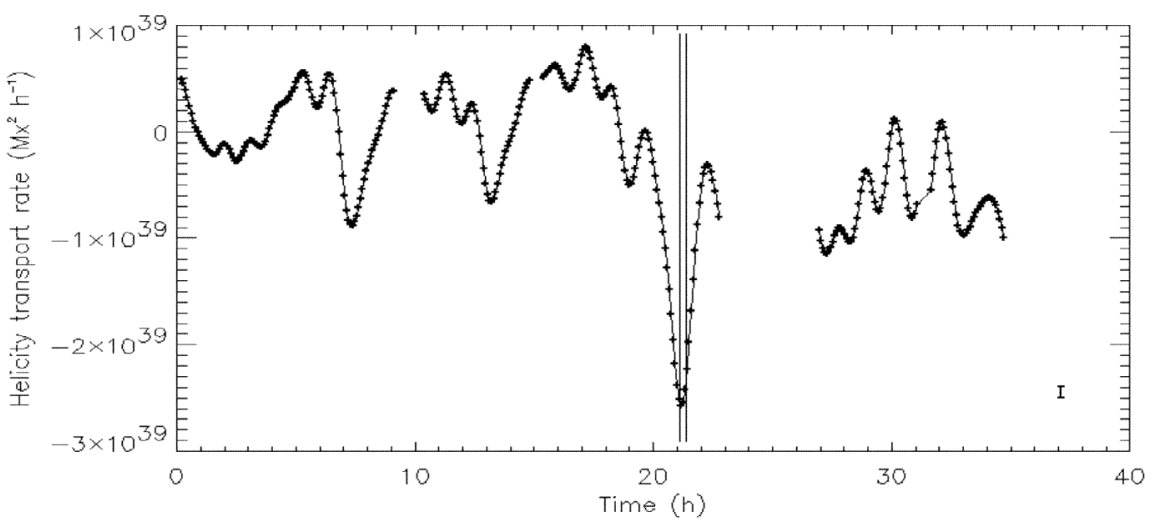

Fig. 7. Helicity transport rate as a function of time in AR 9502. $t=0$ corresponds to 13:00 UT on June 14. The error bar represents the standard deviation of the signal. The vertical lines indicate the start and the end of the flare, as registered by GOES.

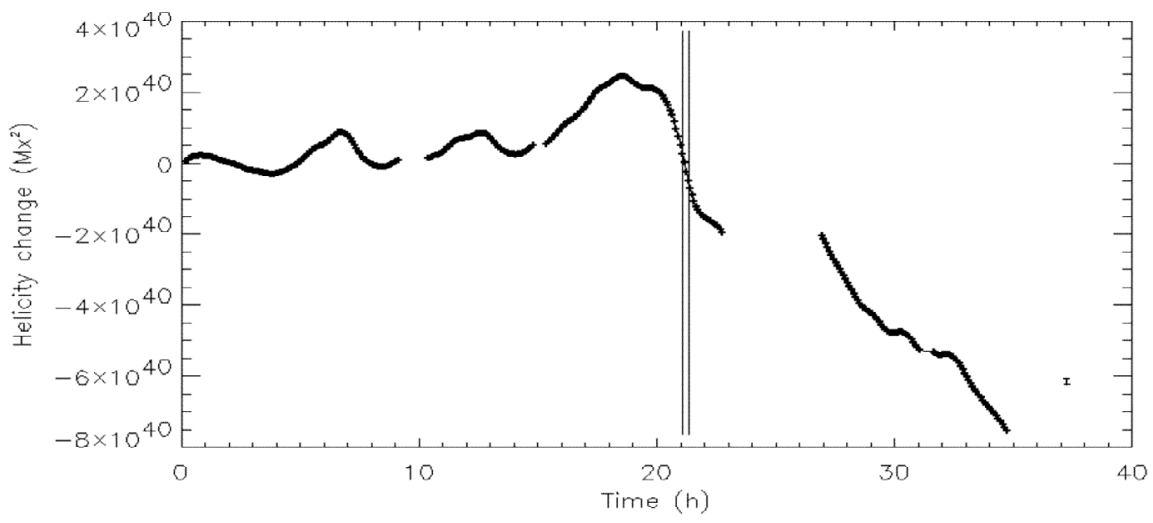

Fig. 8. Accumulated change of the magnetic helicity as a function of time in AR 9502. $t=0$ corresponds to 13:00 UT on June 14. The error bar represents the standard deviation of the signal. The vertical lines indicate the start and the end of the flare.

from the local correlation method, we got the magnetic helicity transport rate with a 5 min time interval in the whole active region. During $17 \mathrm{~h}$ from the beginning of the MDI observations the helicity, besides an apparent oscillation around zero, shows a gradual positive increase, while at 6:00 UT, in coincidence with the observed peculiar motions at filament footpoints, we noted a steep increase in the transport of negative magnetic helicity, reaching $-3 \times 10^{39} \mathrm{Mx}^{2} \mathrm{~h}^{-1}$ at 10:05 UT (Fig. 7). We want to stress that, apart from the negative spikes temporally correlated with three flares registered by GOES and probably due to flare emission affecting magnetograms (HartKorn \& Wang 2004), there is a previous $(1 \leq t \leq 4 \mathrm{~h})$ helicity variation of negative sign before each event. This behaviour suggests that the onset of the filament eruption and the corresponding flare might be associated with the activation of negative helicity injection.

Moreover, the helicity variation is more evident in Fig. 8, where we can see that during the first 18 hours of MDI observations the accumulated helicity change is positive, while during the following $90 \mathrm{~min}$ about $-4 \times 10^{40} \mathrm{Mx}^{2}$ of negative helicity is injected in the corona. During this phase, temporally correlated with the rotating motions at filament footpoints, $T w$ reached the limit for the kink instability and the filament transformed part of its helicity from twist to writhe, starting its eruption. The last two sets of MDI observations (from 15:45 to
23:59 UT) showed a continuous transport of negative helicity with some spikes coincident with two smaller flares registered at 17:27 and 22:42 UT.

Another interesting point is the observed delay in the flare onset from the beginning of the filament eruption. The GOES X-ray flux in $1-8 \AA$ showed that the flare started at 10:01 UT, 8 min after the first signatures of the filament eruption (9:52 UT). This delay allows us to interpret the flare scenario in the context of a typical two-ribbon flare. In fact, before the eruption we noted several unsheared overlying loops which probably provided a downward pull that allowed the filament to maintain a stable configuration. At 9:52 UT the filament lost its stability because of a further increase in the chirality and started to rise. Its rise was followed by the ejection of plasma and by brightenings in most of the active region. Therefore, we think that the twisted rope containing the filament rose slowly during the preflare phase because of the kink instability, meeting the overlying loops. In this scenario the flare triggering should be due to the onset of magnetic reconnection in the arcade enveloping the rising rope (see Priest \& Forbes 2002).

\section{Conclusions}

Recently, an interesting and animated debate about the efficiency of the shear and advection terms for the magnetic 
helicity transport to the corona involved many authors (Chae et al. 2001; Demoulin et al. 2002a,b; Kusano et al. 2002; Moon et al. 2002). The aim of this paper is to provide an observational contribution to this unsolved question.

We analyzed the eruption of a filament located in the active region NOAA 9502. The EUV channel corresponding to the filament was observed by TRACE at $195 \AA$ and showed a sigmoid shape, which indicated a stressed configuration of the magnetic field. Assuming that the filament was contained in a cylindrical curved region anchored at both ends in the photosphere, we found that the geometrical and kinematic parameters were in agreement with the model of the kink instability. We also pointed out the strong temporal correlation between the eruptive flare and a spike in the magnetic helicity transport rate. Moreover, the behavior of the photospheric velocity pattern allowed us to remark the significant role played by the horizontal motions in the helicity injected in the corona and in the onset of solar eruptive flares.

The most important result of this work is therefore provided by the observational evidence that the filament eruption was preceded by horizontal, counterclockwise motions in both areas corresponding to the filament footpoints and that a clear variation in the magnetic helicity change rate, without any significant magnetic flux variation, occurred some hours before the event. This result shows that kink processes may take place by continuous photospheric motions during a time scale of the order of hours. On the other hand, the impulsive variation in the magnetic helicity change rate at the beginning of the filament eruption is in agreement with other observations (Moon et al. 2002; Kusano et al. 2002). This impulsive variation may be a consequence of the solar eruptive flare, that is, a consequence of magnetic suppression or expansion caused by the eruption. In fact, we also noted that the flare ended at 10:20 UT, when the magnetic helicity transport rate returned to its preflare value.

Therefore, we suggest that the balance between the two terms of Eq. (1) depends both on the magnetic configuration and evolution of an active region, although we could infer that the weight of the shearing term is greater in the presence of the $\boldsymbol{S}$ - (or reverse $\boldsymbol{S}$-) shaped filament, indicating a great amount of positive (or negative) magnetic helicity transported to the corona, sufficient to reach an unstable configuration.

Acknowledgements. The authors wish to thank the referee for his useful suggestions and J. Chae for providing the IDL routines for the computation of the magnetic helicity transport rate. Thanks are also due to L. Santagati for the English correction of the manuscript.

\section{References}

Alissandrakis, C. E., Tsiropoula, G., \& Mein, P. 1990, A\&A, 230, 200

Berger, M. A. 1984, Geophys. Astrophys. Fluid. Dynamics, 30, 79

Berger, M. A., \& Field, G. B. 1984, J. Geophys. Res., 147, 133

Berger, M. A., \& Lites, B.W. 2002, Sol. Phys., 213, 213

Chae, J. 2001, ApJ, 560, L95

Chae, J., Wang, H., Qiu, J., et al. 2001, ApJ, 560, 476

Demoulin, P., Mandrini, C. H., van Driel-Gesztelyi, L., et al. 2002a, Sol. Phys., 207, 87

Demoulin, P., Mandrini, C. H., van Driel-Gesztelyi, L., et al. 2002b, A\&A, 382, 650

Demoulin, P., \& Berger, M. A. 2003, Sol. Phys., 215, 203

Hartkorn, K., \& Wang, H. 2004, Sol. Phys., in press

Howard, R. F., Harvey, J. W., \& Forgach, S. 1990, Sol. Phys., 130, 295

Kusano, K., Maeshiro, T., Yokoyama, T., et al. 2002, ApJ, 577, 501

Low, B. C. 1994, Phys. Plasmas, 1(5), 1684

Martin, S. F. 1988, New Perspectives on Solar Prominences, ed. Webb, D., Rust, D., \& Schmieder, B., San Francisco, ASP Conf. Ser., 150,419

Martin, S. F. 2003, Adv. Space Res., 32, 1883

Moon, Y. J., Chae, J., Choe, G. S., et al. 2002, ApJ, 574, 1066

November, L. J. \& Simon, G. W. 1988, ApJ, 333, 427

Priest, E. R., \& Forbes, T. G. 2002, A\&ARv, 10, 313

Romano, P., Contarino, L., \& Zuccarello, F. 2003b, Sol. Phys., 214, 313

Romano, P., Contarino, L., \& Zuccarello, F. 2003a, Sol. Phys., 218, 137

Rust, D. M., \& Kumar, A. 1996, ApJ, 464, L199

Rust, D. M. 2003, Adv. Space Res., 32, 1895

Taylor, J. B. 1986, Rev. Mod. Phys., 58, 741 